\title{
Targeting the Microenvironment in Acute Myeloid Leukemia
}

\author{
Armin Rashidi ${ }^{1}$ and Geoffrey L. Uy ${ }^{1}$ \\ ${ }^{1}$ Section of BMT \& Leukemia, Division of Oncology, Washington University School of Medicine, \\ St. Louis, MO
}

\begin{abstract}
The bone marrow microenvironment plays a critical role in the development, progression, and relapse of acute myeloid leukemia (AML). Similar to normal hematopoietic stem cells, AML blasts express receptors on their surface, allowing them to interact with specific components of the marrow microenvironment. These interactions contribute to both chemotherapy resistance and disease relapse. Preclinical studies and early phase clinical trials have demonstrated the potential for targeting the tumor-microenvironment interactions in AML. Agents currently under investigation include hypoxia-inducible agents and inhibitors of CXCR4 and adhesion molecules such as VLA-4 and E-selectin.
\end{abstract}

\section{Keywords}

leukemia; microenvironment; mesenchymal; niche; resistance; stem cells

\section{Introduction}

\begin{abstract}
The concept of "niche" was first proposed by Schofield [1] to describe the possibility that it is the association between the stem cell and its surrounding cells that determines the stem cell behavior and fate. In adults, hematopoietic stem cells (HSCs) are located in the bone marrow on the endosteal surface in close proximity to osteoblasts and also are associated with vascular endothelial cells located around sinusoidal vessels in the marrow cavity. Within these specialized osteoblast and vascular niches, HSCs interact with a variety of cellular components including mesenchymal stem cells, endothelial cells, osteoblasts, osteoclasts, macrophages, autonomic neurons, and extracellular matrix components including collagen, fibronectin, and laminin fibers. The interaction between HSCs and the niche regulates fundamental HSC cell fate decisions including self-renewal, survival, differentiation, and proliferation.
\end{abstract}

The bone marrow is presumed to be the reservoir for leukemia stem cells (LSCs) which persist after chemotherapy and mediate disease relapse. The interaction of leukemic blasts

Corresponding Author: Geoffrey L. Uy, 660 S. Euclid Ave., Campus Box 8007, Division of Oncology, Washington University School of Medicine, St. Louis, MO 63110, guy@dom.wustl.edu, Tel: 314-747-8439, Fax: 314-454-7551.

Conflicts of interest: Dr. Armin Rashidi and Dr. Geoffrey L. Uy each declare no potential conflicts of interest.

Human and Animal Rights and Informed Consent: This article does not contain any studies with human or animal subjects performed by any of the authors. 
with the bone marrow microenvironment has long been implicated in chemotherapy resistance in AML. Coculture of AML blasts with stromal cell layers inhibits chemotherapyinduced apoptosis in AML; both soluble factors and cell-cell contact mediate chemotherapy resistance [2, 3]. In AML xenotransplantation models into immunodeficient mice, functionally defined AML LSCs are quiescent and home to and engraft within the osteoblast-rich areas of the bone marrow where they are protected from cell cycle-dependent chemotherapy [4]. Microenvironment interactions have also been demonstrated to protect against kinase inhibitors. Extracellular regulated kinase (ERK) activation promotes resistance to FLT 3 inhibition by both direct stromal contact and stroma-derived soluble factors $[5,6]$. In this review, we summarize strategies that are currently being developed to target tumor-microenvironment interactions in AML.

\section{CXCR4 / CXCL12 inhibitors}

The CXCR4/CXCL12 axis is the principal mediator of migration and homing of HSCs to the bone marrow [7]. CXCR4 is a G-protein coupled chemokine receptor expressed on the surface of HSCs. Its ligand, CXCL12, is produced by marrow reticular stromal cells and directs the homing of HSCs to the marrow. The interaction between CXCR4/CXCL12 activates downstream signals resulting in HSC quiescence and regulates the size of the HSC pool [7-9].

Similar to normal HSCs, AML blasts express CXCR4 on their surface and migrate in response to CXCL12 gradients [10, 11]. In AML, higher expression of CXCR4 by CD34 ${ }^{+}$ cells is associated with shorter survival and higher rates of relapse [12, 13]. CXCL12 binding of CXCR4 activates prosurvival and proliferative signaling pathways including the PI3K/Akt and MEK/ERK pathways [14, 15]. CXCR4 inhibitors may physically disrupt the interaction of leukemic cells with the marrow microenvironment by mobilizing cells out of their protective niche. Additionally, these agents can block CXCL12 signaling through CXCR4 which provides critical prosurvival signals to the leukemic cells.

Plerixafor (AMD3100, Mozobil, Sanofi) is a bicyclam small molecule inhibitor of CXCR4. The drug is approved in both the United States and Europe for the mobilization of autologous hematopoietic stem and progenitor cells in patients with multiple myeloma and non-Hodgkin lymphoma [16, 17]. In cell lines, plerixafor blocks the protective effects of stromal cells against chemotherapy in AML cell lines. Using a murine model of acute promyelocytic leukemia, the addition of plerixafor to cytarabine decreased the leukemia burden and improved overall survival (OS) compared to cytarabine alone [18]. Plerixafor also inhibits the proliferation of AML cell lines and induces gene expression changes that mimic granulocye differentiation [19]. A related CXCR4 antagonist, AMD3465, downregulates CXCR4 phosphorylation and suppresses stroma-activated survival pathways (PI3K/Akt and MEK/ERK) in FLT3-mutated AML cells. The stroma is known to activate two prosurvival pathways (PI3K/Akt and MEK/ERK) in AML cells with FLT3 mutation, and confer resistance to both chemotherapy and FLT3 inhibition [14]. In murine xenograft models, AMD3465 mobilized FLT3-mutated AML cells into the peripheral blood, eliminated the stroma-mediated support of the microenvironment, and made them more susceptible to cytarabine or the FLT3 inhibitor sorafenib [14]. 
In the initial phase I/II study of plerixafor as a chemosensitizing agent, 52 patients with relapsed or refractory AML were treated with a combination of plerixafor and chemotherapy (mitoxantrone, etoposide and cytarabine) [20]. The regimen was safe and associated with an overall complete remission and complete remission with incomplete count recovery (CR/ CRi) rate of $46 \%$. A two-fold increase in AML blast mobilization into the peripheral blood was observed [20]. Plerixafor has also been investigated in a phase I clinical trial with newly diagnosed AML patients in combination with cytarabine and daunorubicin. The regimen was comparable in its toxicity profile to standard chemotherapy and $67 \%$ of patients achieved a CR [21]. A subsequent study in AML have combined plerixafor with G-CSF due to its known synergistic activities for stem cell mobilization and the ability of G-CSF to downregulate CXCL12 expression [22]. Additional studies have combined plerixafor with decitabine in older adults with AML with a $43 \% \mathrm{CR} / \mathrm{CRi}$ rate and a median response duration of 4.5 months [23]. A combination of plerixafor, sorafenib, and G-CSF in FLT3mutated patients yielded an overall response rate of 77\% [24]. In all studies, plerixafor has been found to mobilize AML blasts into the peripheral circulation. In addition, upregulation of surface CXCR4 on AML blasts has been observed following exposure to plerixafor [24]. Plerixafor appears to be safe and well tolerated without evidence of either delayed hematopoietic recovery or symptoms of hyperleukocytosis.

Additional CXCR4 inhibitors have been tested in both preclinical models of tumor sensitization and clinical trials for AML. Ulocuplumab (BMS-936564/MDX-1338, Bristol Myers Squibb) is a fully human IgG4 monoclonal antibody to CXCR4. Compared to plerixafor, ulocuplumab possesses longer half-life resulting in more sustained CXCR4 inhibition and also demonstrates antitumor activity alone by inducing apoptosis in tumor cells [25]. In a phase I clinical trial of ulocuplumab in combination with chemotherapy (mitoxantrone, etoposide and cytarabine) in patients with relapsed/refractory AML the overall CR/CRi rate was $51 \%$ with a median 2- and 5-fold mobilization of leukocytes and leukemic blasts into the peripheral circulation at day 8 [26]. Other CXCR4 inhibitors in clinical development either for stem cell mobilization or tumor sensitization include BL-8040 (BioLineRx), POL6326 (Polyphor), LY2510924 (Eli Lilly) and olaptesed pegol (NOX-A12, NOXXON PharmaAg) [27-29].

\section{Very late antigen-4 (VLA-4)}

Very late antigen-4 (VLA-4) is a member of the integrin family of cell surface adhesion molecules. VLA- 4 is a heterodimer comprised of the $a 4$ and $\beta 1$ integrin subunits and is expressed on leukocytes. VLA-4 mediates retention of HSCs within the marrow through binding of vascular cell adhesion molecule-1 (VCAM-1) expressed by osteoblasts and endothelial cells and by binding to an extracellular matrix component, fibronectin. Activation of VLA-4 enhances HSC adhesion to the endothelial cells which is followed by their transendothelial migration [30].

VLA-4 is variably expressed on AML blasts. Clinically, high expression of VLA-4 by AML cells is associated with a reduced risk of relapse in children [31]. In a study of adult patients treated on SWOG trials, VLA-4 expression was not associated with outcome, but increased binding of soluble VCAM-1 via VLA-4 was associated with longer OS [32]. The interaction 
between VLA-4 on AML cells and fibronectin on stromal cells activates the PI3K/Akt/Bcl-2 signaling pathway and mediates resistance to chemotherapy [33]. In preclinical models, a VLA-4 antibody was able to overcome resistance to cytarabine-mediated by attachment of VLA-4 to fibronectin in a U937 leukemia cell line [33].

A humanized monoclonal antibody against VLA-4, natalizumab (Tysabri), is in clinical use for the treatment of autoimmune diseases including multiple sclerosis and Crohn's disease. In a xenograph model of primary AML cells engrafted in NOD/SCIDIL2R $\gamma$ mice, natalizumab-treated mice survived longer than untreated animals [34]. In patients with multiple sclerosis treated with natalizumab, an increase in HSCs in the peripheral circulation for at least a month after administration has been observed [35]. Natalizumab is associated with JC virus-associated progressive multifocal leukoencephalopathy which is likely a direct result of its prolonged effects on lymphocyte trafficking and may limit its clinical utility as a chemosensitizing agent in AML [36].

Alternative agents that block VLA-4 are in clinical development. AS 101 (ammonium trichloro (dioxoethylene-o,o') tellurate) is a compound that results in redox inhibition of VLA-4 after binding to fibronectin. AS101 triggered cytoskeletal conformational changes that decreased PI3K/Akt/Bcl2 signaling abrogated drug resistance and prolonged survival in mice receiving chemotherapy [37]. Clinical trials are currently being planned in patients with AML and MDS.

\section{E-selectin}

E-selectin is a cell adhesion molecule which mediates the tethering and rolling of leukocytes to vascular endothelial cells. Glycoproteins including CD44 and E-selectin ligand-1 (ESL-1) function as the ligands for E-selectin on HSCs and mediate proliferation and exit of HSCs from the quiescent state [38]. Genetic deletion of E-selectin or administration of an Eselectin antagonist in mice enhances HSC quiescence and self-renewal potential and improves HSC survival following treatment with chemotherapy or radiation [38].

CD44 is a glycoprotein which has important roles in cell adhesion and migration. CD44 functions as the main receptor for hyaluronic acid. CD44 splice variants are expressed both on HSC and AML blasts, allowing it to bind to E-selelctin and L-selectin. A monoclonal antibody directed against CD44 blocked engraftment of primary AML cells into NOD/SCID mice [39]. Expression levels of E-selectin ligands are higher in the blasts of patients with relapsed/refractory AML disease compared to de novo disease. Adhesion of blasts expressing E-selectin ligands to the vascular niche enhances blast survival by activating the Wnt signaling pathway [40]. A specific E-selectin small molecule inhibitor, GMI-1271, is currently in clinical development for hematologic malignancies including AML. Blockade of E-selectin with GMI-1271 enhanced the effect of daunorubicin and cytarabine, and reduced the leukemia burden in a xenograft model [40].

\section{Bone marrow hypoxia}

Compared to HSCs in the normal bone marrow, AML cells exist in the marrow under hypoxic conditions. Preferential localization in hypoxic niches minimizes LSC exposure to 
chemotherapeutic drugs. LSCs are better adapted than normal HSCs in expanded, hypoxic marrow niches [41, 42]. Hypoxia within the microenvironment has pivotal prosurvival effects on AML cells via activation of the PI3K/Akt/mTOR pathway and Pim-1 expression $[43,44]$. Hypoxia-induced mTOR activation occurs via PI3K/Akt-dependent and independent mechanisms [45, 46]. mTOR mediates downstream signaling of PI3K/Akt by increased Akt phosphorylation [47]. The latter effect is mediated by mTOR complexes mTORCl and mTORC2 [48, 49]. Hypoxia-inducible factor (HIF) $1 \mathrm{a}$ also directly upregulates the expression of TGF $\beta 1$ [50], which in turn increases the expression of CXCR4 on blasts [51,52]. While the interaction between CXCL12 and CXCR4 activates the intrinsic pathway of apoptosis in AML cells in vitro [53], the marrow microenvironment protects AML cells against CXCR4/CXCL12-induced apoptosis by an unknown mechanism [54]. Finally, tissue hypoxia may limit the recruitment of the immune cells which can exert anti-leukemic effects [41, 42].

TH-302 is a 2-nitroimidazole-linked prodrug which under hypoxic conditions releases the DNA alkylating agent bromo-isophohoramide mustard. After exposure to hypoxia, previously resistant AML cells became sensitive to cytarabine and underwent apoptosis in the presence of TH-302. This was associated with decreased expression of HIF-1a, cell cycle arrest, and DNA strand breaks [55]. In a phase I studies of TH-302 in 39 patients with refractory AML, transient cytoreduction was observed in most patients with one CR and once CRi [56].

\section{Conclusions}

With recent large scale DNA sequencing studies, it is evident that significant heterogeneity exists between and even within individual cases of AML. Strategies that target specific mutations in AML may only apply to small, select group of patients with AML and may even only target individual subclones within a patient. Therefore, targeting tumor microenvironment interactions is an attractive strategy to improve the outcome of AML in a non-cell autonomous fashion. Mechanisms of environment-mediated drug resistance cross a number of hematologic malignancies with similar strategies being pursued in multiple myeloma, chronic lymphocytic leukemia and even solid tumors. While many of the mechanisms regarding tumor microenvironment may be shared between different hematologic malignancies, others may be unique. For examples, expression of the parathyroid hormone receptor in osteoblasts attenuates CML but enhances AML in mouse transplantation models [57]. Ongoing studies will elucidate the potential of targeting the bone marrow microenvironment in improving the outcome of patients with AML.

\section{References}

1. Schofield R. The relationship between the spleen colony-forming cell and the haemopoietic stem cell. Blood Cells. 1978; 4(1-2):7-25. [PubMed: 747780]

2. Bendall LJ, et al. Bone marrow adherent layers inhibit apoptosis of acute myeloid leukemia cells. Exp Hematol. 1994; 22(13):1252-60. [PubMed: 7957711]

3. Garrido SM, et al. Acute myeloid leukemia cells are protected from spontaneous and drug-induced apoptosis by direct contact with a human bone marrow stromal cell line (HS-5). Exp Hematol. 2001; 29(4):448-57. [PubMed: 11301185] 
4. Ishikawa F, et al. Chemotherapy-resistant human AML stem cells home to and engraft within the bone-marrow endosteal region. Nat Biotechnol. 2007; 25(11):1315-21. [PubMed: 17952057]

5. Yang X, Sexauer A, Levis M. Bone marrow stroma-mediated resistance to FLT3 inhibitors in FLT3-ITD AML is mediated by persistent activation of extracellular regulated kinase. Br J Haematol. 2014; 164(1):61-72. [PubMed: 24116827]

6. Sexauer A, et al. Terminal myeloid differentiation in vivo is induced by FLT3 inhibition in FLT3/ITD AML. Blood. 2012; 120(20):4205-14. [PubMed: 23012328]

7. Sugiyama T, et al. Maintenance of the hematopoietic stem cell pool by CXCL12-CXCR4 chemokine signaling in bone marrow stromal cell niches. Immunity. 2006; 25(6):977-88. [PubMed: 17174120]

8. Omatsu Y, et al. The essential functions of adipo-osteogenic progenitors as the hematopoietic stem and progenitor cell niche. Immunity. 2010; 33(3):387-99. [PubMed: 20850355]

9. Greenbaum A, et al. CXCL12 in early mesenchymal progenitors is required for haematopoietic stem-cell maintenance. Nature. 2013; 495(7440):227-30. [PubMed: 23434756]

10. Mohle R, et al. The chemokine receptor CXCR-4 is expressed on CD34+ hematopoietic progenitors and leukemic cells and mediates transendothelial migration induced by stromal cellderived factor-1. Blood. 1998; 91(12):4523-30. [PubMed: 9616148]

11. Mohle R, et al. Functional response of leukaemic blasts to stromal cell-derived factor-1 correlates with preferential expression of the chemokine receptor CXCR4 in acute myelomonocytic and lymphoblastic leukaemia. Br J Haematol. 2000; 110(3):563-72. [PubMed: 10997965]

12. Rombouts EJ, et al. Relation between CXCR-4 expression, Flt3 mutations, and unfavorable prognosis of adult acute myeloid leukemia. Blood. 2004; 104(2):550-7. [PubMed: 15054042]

13. Spoo AC, et al. CXCR4 is a prognostic marker in acute myelogenous leukemia. Blood. 2007; 109(2):786-91. [PubMed: 16888090]

14. Zeng Z, et al. Targeting the leukemia microenvironment by CXCR4 inhibition overcomes resistance to kinase inhibitors and chemotherapy in AML. Blood. 2009; 113(24):6215-24. [PubMed: 18955566]

$15^{*}$. Chen Y, et al. CXCR4 downregulation of let-7a drives chemoresistance in acute myeloid leukemia. J Clin Invest. 2013; 123(6):2395-407. This study demonstrated that CXCR4-mediated downregulation of the microRNA let-7a drives chemoresistance in an AML cell line. Xenograft mice of human AML cell lines engineered to overexpress let-7a were more chemosensitive and showed improved survival. [PubMed: 23676502]

16. DiPersio JF, et al. Phase III prospective randomized double-blind placebo-controlled trial of plerixafor plus granulocyte colony-stimulating factor compared with placebo plus granulocyte colony-stimulating factor for autologous stem-cell mobilization and transplantation for patients with non-Hodgkin's lymphoma. J Clin Oncol. 2009; 27(28):4767-73. [PubMed: 19720922]

17. DiPersio JF, et al. Plerixafor and G-CSF versus placebo and G-CSF to mobilize hematopoietic stem cells for autologous stem cell transplantation in patients with multiple myeloma. Blood. 2009; 113(23):5720-6. [PubMed: 19363221]

18. Nervi B, et al. Chemosensitization of acute myeloid leukemia (AML) following mobilization by the CXCR4 antagonist AMD3100. Blood. 2009; 113(24):6206-14. [PubMed: 19050309]

19. Tavor S, et al. The CXCR4 antagonist AMD3100 impairs survival of human AML cells and induces their differentiation. Leukemia. 2008; 22(12):2151-5158. [PubMed: 18769446]

20**. Uy GL, et al. A phase 1/2 study of chemosensitization with the CXCR4 antagonist plerixafor in relapsed or refractory acute myeloid leukemia. Blood. 2012; 119(17):3917-24. This is the largest clinical trial to date of chemosensitization by mobilization using plerixafor (a CXCR4 inhibitor) in patients with relapsed or refractory AML. The study demonstrated safety of the combination therapy (plerixafor and chemotherapy) with an overall complete remission and complete remission with incomplete count recovery (CR/CRi) rate of 46\%. [PubMed: 22308295]

21. Uy GL, et al. Safety and tolerability of plerixafor in combination with cytarabine and daunorubicin in patients with newly diagnosed acute myeloid leukemia- preliminary results from a phase I study. Blood. 2011

22. Petit I, et al. G-CSF induces stem cell mobilization by decreasing bone marrow SDF-1 and upregulating CXCR4. Nat Immunol. 2002; 3(7):687-94. [PubMed: 12068293] 
23. Roboz, GJ., et al. 55th Ameican Society of Hematology Annual Meeting and Exposition. New Orleans, LA: Blood; 2013. Combining decitabine with plerixafor yields a high response rate in newly diagnosed older patients with AML.

24. Andreeff M, et al. Mobilization and elimination of FLT3-ITD+ acute myelogenous leukemia (AML) stem/progenitor cells by plerixafor/G-CSF/sorafenib: results from a phase 1 trial in relapsed/refractory AML patients. Blood. 2012:120.

25. Kuhne MR, et al. BMS-936564/MDX-1338: a fully human anti-CXCR4 antibody induces apoptosis in vitro and shows antitumor activity in vivo in hematologic malignancies. Clinical cancer research : an official journal of the American Association for Cancer Research. 2013; 19(2):357-66. [PubMed: 23213054]

26. Becker PS, et al. Targeting the CXCR4 pathway: safety, tolerability and clinical activity of Ulocuplumab (BMS-936564), an anti-CXCR4 antibody, in relapsed/refractory acute myeloid leukemia. Blood. 2014

27. Galsky MD, et al. A phase I trial of LY2510924, a CXCR4 peptide antagonist, in patients with advanced cancer. Clinical cancer research : an official journal of the American Association for Cancer Research. 2014; 20(13):3581-8. [PubMed: 24727324]

28. Marasca R, Maffei R. NOX-A12: mobilizing CLL away from home. Blood. 2014; 123(7):952-3. [PubMed: 24526776]

29. Hoellenriegel J, et al. The Spiegeimer NOX-A12, a novel CXCL12 inhibitor, interferes with chronic lymphocytic leukemia cell motility and causes chemosensitization. Blood. 2014; 123(7): 1032-9. [PubMed: 24277076]

30. Peled A, et al. The chemokine SDF-1 activates the integrins LFA-1, VLA-4, and VLA-5 on immature human CD34(+) cells: role in transendothelial/stromal migration and engraftment of NOD/SCID mice. Blood. 2000; 95(11):3289-96. [PubMed: 10828007]

31. Walter RB, et al. High expression of the very late antigen-4 integrin independently predicts reduced risk of relapse and improved outcome in pediatric acute myeloid leukemia: a report from the children's oncology group. J Clin Oncol. 2010; 28(17):2831-8. [PubMed: 20421533]

32. Becker PS, et al. Very late antigen-4 function of myeloblasts correlates with improved overall survival for patients with acute myeloid leukemia. Blood. 2009; 113(4):866-74. [PubMed: 18927435]

33. Matsunaga T, et al. Interaction between leukemic-cell VLA-4 and stromal fibronectin is a decisive factor for minimal residual disease of acute myelogenous leukemia. Nat Med. 2003; 9(9):1158-65. [PubMed: 12897778]

34. Jiang E, et al. VLA4 Blockade In Acute Myeloid Leukemia. 2013; 122:3944-3944.

35. Bonig $\mathrm{H}$, et al. Increased numbers of circulating hematopoietic stem/progenitor cells are chronically maintained in patients treated with the CD49d blocking antibody natalizumab. Blood. 2008; 111(7):3439-41. [PubMed: 18195093]

36. Bloomgren $\mathrm{G}$, et al. Risk of natalizumab-associated progressive multifocal leukoencephalopathy. N Engl J Med. 2012; 366(20):1870-80. [PubMed: 22591293]

37. Layani-Bazar A, et al. Redox modulation of adjacent thiols in VLA-4 by ASI01 converts myeloid leukemia cells from a drug-resistant to drug-sensitive state. Cancer Res. 2014; 74(11):3092-103. [PubMed: 24699624]

38**. Winkler LG, et al. Vascular niche E-selectin regulates hematopoietic stem cell dormancy, self renewal and chemoresistance. Nat Med. 2012; 18(11):1651-7. This study demonstrated in mice that E-selectin, expressed on the endothelial cells in the vascular HSC niche, promoted HSC proliferation and exit from the quiescent state, whereas E-selectin antagonism protected HSCs against chemotherapy and irradiation. These results highlight the importance of the microenvironment in HSC fate decisions. [PubMed: 23086476]

39. Jin L, et al. Targeting of CD44 eradicates human acute myeloid leukemic stem cells. Nat Med. 2006; 12(10):1167-74. [PubMed: 16998484]

40. Chien S, et al. Adhesion of acute myeloid leukemia blasts to E-selectin in the vascular niche enhances their survival by mechanisms such as Wnt activation. Blood. 2013; 122:2161. 
41. Jensen PO, et al. Increased cellular hypoxia and reduced proliferation of both normal and leukaemic cells during progression of acute myeloid leukaemia in rats. Cell Prolif. 2000; 33(6): 381-95. [PubMed: 11101010]

42. Giuntoli S, et al. Severe hypoxia defines heterogeneity and selects highly immature progenitors within clonal erythroleukemia cells. Stem Cells. 2007; 25(5):1119-25. [PubMed: 17255519]

43. Konopleva MY, Jordan CT. Leukemia stem cells and microenvironment: biology and therapeutic targeting. J Clin Oncol. 2011; 29(5):591-9. [PubMed: 21220598]

44. Kornblau SM, et al. Simultaneous activation of multiple signal transduction pathways confers poor prognosis in acute myelogenous leukemia. Blood. 2006; 108(7):2358-65. [PubMed: 16763210]

45. Chen EY, et al. Hypoxia activates a platelet-derived growth factor receptor/phosphatidylinositol 3 kinase/Akt pathway that results in glycogen synthase kinase-3 inactivation. Cancer Res. 2001; 61(6):2429-33. [PubMed: 11289110]

46. Vasseur S, et al. DJ-I/PARK7 is an important mediator of hypoxia-induced cellular responses. Proc Natl Acad Sci USA. 2009; 106(4):1111-6. [PubMed: 19144925]

47. Sun HL, et al. YC-1 inhibits HIF-1 expression in prostate cancer cells: contribution of Akt/NFkappaB signaling to HIF-1 alpha accumulation during hypoxia. Oncogene. 2007; 26(27):3941-51. [PubMed: 17213816]

48. Kim DH, et al. mTOR interacts with raptor to form a nutrient-sensitive complex that signals to the cell growth machinery. Cell. 2002; 110(2):163-75. [PubMed: 12150925]

49. Sarbassov DD, et al. Rictor, a novel binding partner of mTOR, defines a rapamycin-insensitive and raptor-independent pathway that regulates the cytoskeleton. Curr Biol. 2004; 14(14):1296-302. [PubMed: 15268862]

50. Zhang H, et al. Cellular response to hypoxia involves signaling via Smad proteins. Blood. 2003; 101(6):2253-60. [PubMed: 12411310]

51. Buckley CD, et al. Persistent induction of the chemokine receptor CXCR4 by TGF-beta 1 on synovial $\mathrm{T}$ cells contributes to their accumulation within the rheumatoid synovium. J Immunol. 2000; 165(6):3423-9. [PubMed: 10975862]

52. Katoh M, Katoh M. Integrative genomic analyses of CXCR4: transcriptional regulation of CXCR4 based on TGFbeta, Nodal, Activin signaling and POU5F1, FOXA2, FOXC2, FOXH1, SOX17, and GFI1 transcription factors. Int J Oncol. 2010; 36(2):415-20. [PubMed: 20043076]

53. Kremer KN, et al. CXCR4 chemokine receptor signaling induces apoptosis in acute myeloid leukemia cells via regulation of the Bcl-2 family members Bcl-XL, Noxa, and Bak. J Biol Chem. 2013; 288(32):22899-914. [PubMed: 23798675]

54. Kremer KN, et al. Osteoblasts protect AML cells from SDF-1-induced apoptosis. J Cell Biochem. 2014; 115(6):1128-37. [PubMed: 24851270]

$55^{*}$. Portwood S, et al. Activity of the hypoxia-activated prodrug, TH-302, in preclinical human acute myeloid leukemia models. Clin Cancer Res. 2013; 19(23):6506-19. TH-302 is a 2nitroimidazole-linked prodrug which under hypoxic conditions releases an alkylating agent. In this study, TH-302 made chemoresistant human AML cells chemosensitive under hypoxia, demonstrating the role of hypoxia in AML chemoresistance. [PubMed: 24088735]

56. Handisides DR, et al. A Phase 1 Study Of TH-302, An Investigational Hypoxia-Targeted Drug, In Patients With Advanced Leukemias. 2013; 122:3920-3920.

57. Krause DS, et al. Differential regulation of myeloid leukemias by the bone marrow microenvironment. Nat Med. 2013; 19(11):1513-7. [PubMed: 24162813] 


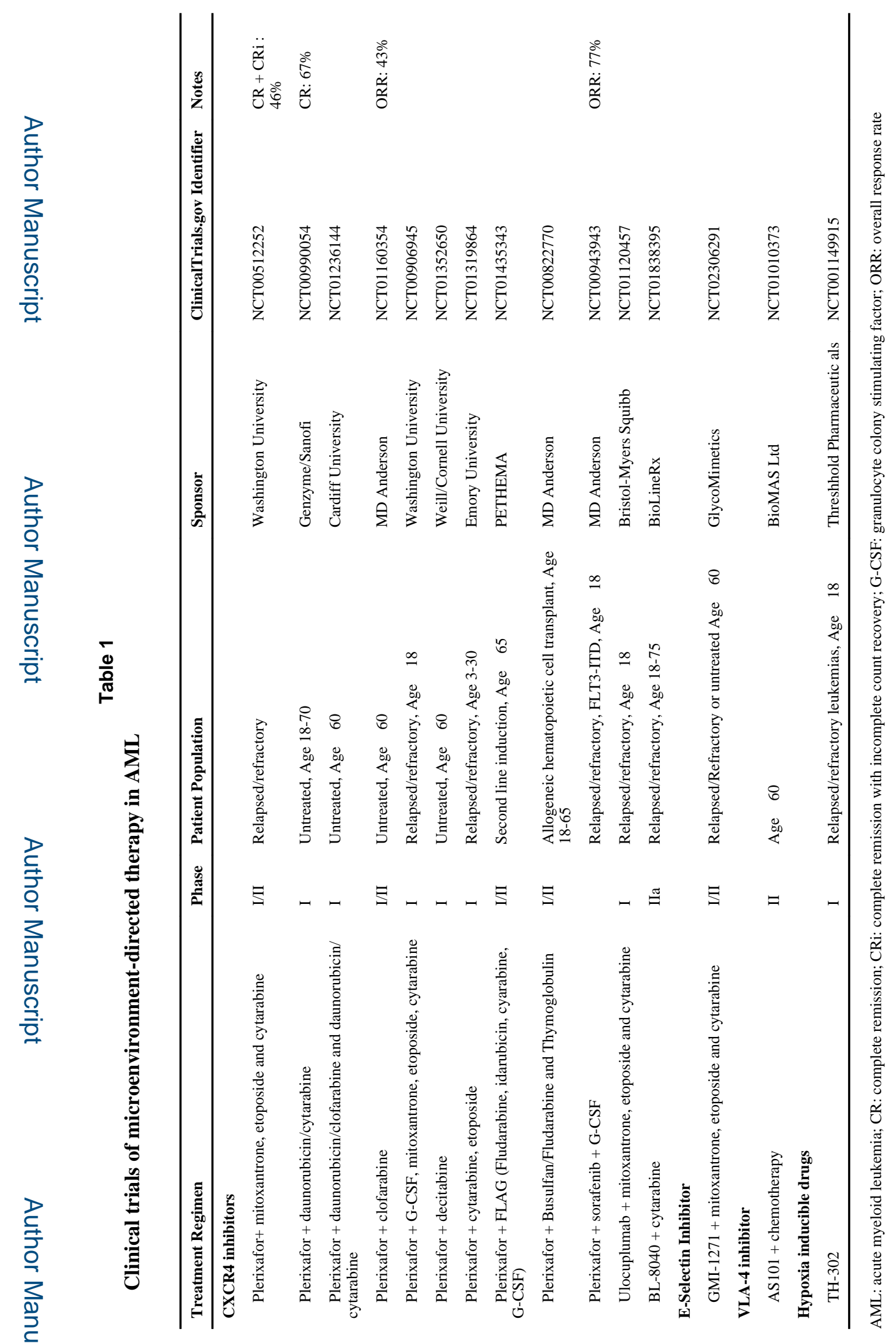

Curr Hematol Malig Rep. Author manuscript; available in PMC 2016 June 01. 